\title{
Non-strange and strange pentaquarks with hidden charm
}

\author{
V.V. Anisovich ${ }^{+}$, M.A. Matveev ${ }^{+}$, J. Nyiri*, A.V. Sarantsev ${ }^{+}$, A.N. Semenova ${ }^{+}$ \\ June 22, 2021 \\ + National Research Centre "Kurchatov Institute": Petersburg Nuclear Physics Institute, \\ Gatchina, 188300, Russia \\ $\diamond$ Helmholtz-Institut für Strahlen- und Kernphysik, Universität Bonn, Germany \\ ${ }^{*}$ Institute for Particle and Nuclear Physics, Wigner RCP, Budapest 1121, Hungary
}

\begin{abstract}
Non-strange and strange pentaquaks with hidden charm are considered as diquarkdiquark-antiquark composite systems. Spin and isospin content of such exotic states is discussed and masses are evaluated.
\end{abstract}

Keywords: Quark model; diquark; exotic states.

PACS numbers: 12.40.Yx, 12.39.-x, 14.40.Lb

\section{Introduction}

The world of hadrons is a world of composite particles. The idea of quark structure of hadrons appeared long ago:1 mesons are quark-antiquark systems, baryons are built of three quarks. This structure provides a possibility for the systematization of the hadrons and model calculation of the hadron characteristics.

For many decades this procedure worked perfectly, and it is still so for the mesons. The mesonic sector of light quarks $(u, d, s)$ fits well the flavours, the mesons are placed with a good accuracy on the Regge trajectories and the trajectories of radial excitations. ${ }^{2}$

However, the growing number of experimental results leads to unexpected problems in the baryonic sector. It turned out that much less baryons were detected experimentally than the number of predicted three-quark excitations.

Introduction of diquarks as pimary constituents for hadrons can improve this contradictory situation (the notion of the diquark was introdused also by Gell-Mann ${ }^{\mathbb{1}}$ ). If diquarks exist and can be considered as primary objects, the number of predicted baryon states has to be smaller than in the three-quark scheme. In the framework of such scheme of baryons $s^{31}$ "towers" of baryonic states appear, i.e. groups of resonances with the same masses but different spins. The existence of such towers could give an explanation for the absence of signals coming from 
groups of resonances which are shielded by their "neighbours". Unfortunately, there are no experimental results demonstrating the existence of such groups of states. This may, however, not confirm their absence since there was no real search for towers.

The scheme with constituent quarks and diquarks suggests an existence diquark-antidiquark mesons and diquark-diquark-antiquark baryons.

If the signal detected by the LHCb collaboration ${ }^{4}$ in the spectrum $p J / \psi$ of the reaction $\Lambda \rightarrow$ $K^{-}(p J / \psi)$ is inducted by the pole singularity of the amplitude, this is a definite confirmation of the fact that the baryon sector consists not only of excitations of three-quark or quark-diquark degrees of freedom, but also of a growing number of constituents.

Such a possibility for the growth of the hadron sector was considered already long ago, ${ }^{[5+7}$ and the vivid discussion continues up to now for both the case of mesons ${ }^{8-12}$ and baryons. 13

In a world of particles built of diquarks we arrived at a point where we are able to define the quantum numbers of some new hadrons and estimate the intervals between their probable masses. In the present paper we try to do this on the basis of the systematization of mesons $s^{15}$ and baryons. 16

In terms of the quark-diquark states the $\mathrm{LHCb}$ pentaquark ${ }^{4}$ can be presented as a threebody system $\bar{c} \cdot(c u) \cdot(u d)$ where $(c u)$ and $(u d)$ refer to heavy-light and light-light diquarks. A diquark is a color antitriplet member, similar to a antiquark, and the three-body system diquark-diquark-antiquark has a color structure similar to that in low-lying baryons. It is reasonable to suppose a similarity of color forces in three-quark and diquark-diquark-antiquark systems.

The LHCb collaboration ${ }^{4}$ within study $\Lambda_{b}^{0} \rightarrow K^{-} J / \psi p$ decay presents two candidates for pentaquarks decaying to the $p J / \psi$-channel. That are a narrow peak $5 / 2^{?}(4450 \pm 4)$ with a width $\Gamma=(39 \pm 24) \mathrm{MeV}$ and a broad state $3 / 2^{?}(4380 \pm 38)$ with $\Gamma=(205 \pm 94) \mathrm{MeV}$, the opposite parity of the states is preferred. In Ref. 17 the variant of $3 / 2^{-}$and $5 / 2^{+}$is accepted, in Ref. 16 we consider a version with $3 / 2^{+}$and $5 / 2^{-}$. Actually we suppose that the narrow peak $5 / 2^{-}(4450 \pm 4)$ is a genuine $\mathrm{S}$-wave pentaquark while in the mass region $M_{p J / \psi} \simeq 4380 \mathrm{MeV}$ other subruns can contribute thus imitating the broad state. That are final state rescatterings of outgoing hadrons and/or reflection of possible exotic meson resonances from the $K^{-} J / \psi$ channel. The matter is the S-wave diquark-antidiquark states with open strangeness which have masses in the range of $4000-4550 \mathrm{MeV} \sqrt{15}$ and can be produced in the $K^{-} J / \psi$ spectrum.

The LHCb pentaquark states are now a subject of wide discussion. 19 we continue the discussion presenting possible classification of the non-strange and strange pentaquarks with hidden charm and estimate their masses.

\section{Pentaquarks as diquark-diquark-antiquark systems}

We discuss a scheme in which the exotic states are formed by standard QCD-motivated interactions (gluonic exchanges, confinement forces) but with diquarks as constituents. In the color space we write for the pentaquark:

$$
\begin{aligned}
& P_{\bar{c} c u u d}^{+}=\epsilon_{\alpha \beta \gamma} \bar{c}^{\alpha}(c u)^{\beta}(u d)^{\gamma}+\text { permutations of the } u, d \text { quarks } \\
& (c u)^{\beta}=\epsilon^{\beta \beta^{\prime} \gamma^{\prime}} c_{\beta^{\prime}} u_{\gamma^{\prime}}, \quad(u d)^{\gamma}=\epsilon^{\gamma \beta^{\prime \prime} \gamma^{\prime \prime}} u_{\beta^{\prime \prime}} d_{\gamma^{\prime \prime}},
\end{aligned}
$$


where $\alpha, \beta, \gamma$ refer to color indices.

\subsubsection{Spin structure of the pentaquarks}

We work with two diquarks, scalar $S\left(0^{+}\right)$one and axial-vector $A\left(1^{+}\right)$one. In terms of these diquarks the color-flavor wave function of pentaquark reads:

$$
P_{\bar{c} \cdot(c q)\left(q^{\prime} q^{\prime \prime}\right)}=\bar{c}^{\alpha} \cdot \epsilon_{\alpha \beta \gamma}\left|\begin{array}{c}
S_{(c q)}^{\beta} \\
A_{(c q)}^{\beta}
\end{array}\right| \cdot\left|\begin{array}{c}
S_{\left(q^{\prime} q^{\prime \prime}\right)}^{\gamma} \\
A_{\left(q^{\prime} q^{\prime \prime}\right)}^{\gamma}
\end{array}\right|, \quad \text { with } q, q^{\prime}, q^{\prime \prime}=u, d, s .
$$

It results in six diquark-diquark states:

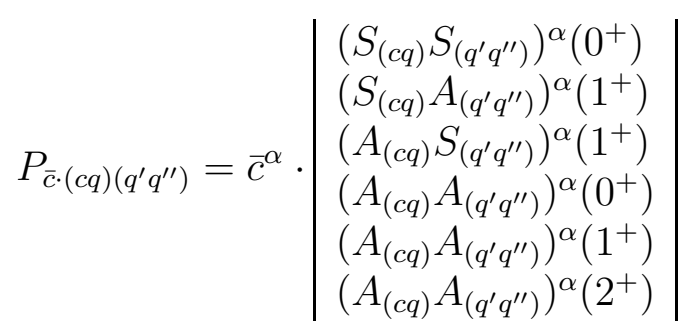

with the spin-parity numbers for diquark-diquark subsystems equal to $J^{P}=0^{+}, 1^{+}, 2^{+}$.

\subsubsection{Isospin structure of the pentaquarks}

We have the following isospin-spin structure for the diquarks:

$$
\begin{array}{ll}
S_{(c q)}(I=1 / 2, J=0), & A_{(c q)}(I=1 / 2, J=1), \\
S_{\left(q^{\prime} q^{\prime \prime}\right)}(I=0, J=0), & A_{\left(q^{\prime} q^{\prime \prime}\right)}(I=1, J=1),
\end{array}
$$

and the isospin-spin sector of the pentaquarks $P\left(I, J^{P}\right)$ reads:

$$
\begin{aligned}
& \bar{c} S_{(c q)} S_{\left(q^{\prime} q^{\prime \prime}\right)}\left(0^{+}\right): \quad P\left(\frac{1}{2}, \frac{1}{2}^{-}\right), \\
& \bar{c} S_{(c q)} A_{\left(q^{\prime} q^{\prime \prime}\right)}\left(1^{+}\right): \quad P\left(\frac{1}{2}, \frac{1}{2}^{-}\right), P\left(\frac{1}{2}, \frac{3}{2}^{-}\right), P\left(\frac{3}{2}, \frac{1}{2}^{-}\right), P\left(\frac{3}{2}, \frac{3}{2}^{-}\right) \text {, } \\
& P\left(I, J^{P}\right)=\begin{array}{ll}
\bar{c} A_{(c q)} S_{\left(q^{\prime} q^{\prime \prime}\right)}\left(1^{+}\right): & P\left(\frac{1}{2}, \frac{1}{2}^{-}\right), P\left(\frac{1}{2}, \frac{3}{2}^{-}\right), \\
& \bar{c} A_{(c q)} A_{\left(q^{\prime} q^{\prime \prime}\right)}\left(0^{+}\right): P\left(\frac{1}{2}, \frac{1}{2}^{-}\right), P\left(\frac{3}{2}, \frac{1}{2}^{-}\right),
\end{array} \\
& \bar{c} A_{(c q)} A_{\left(q^{\prime} q^{\prime \prime}\right)}\left(1^{+}\right): \quad P\left(\frac{1}{2}, \frac{1}{2}^{-}\right), P\left(\frac{1}{2}, \frac{3}{2}^{-}\right), P\left(\frac{3}{2}, \frac{1}{2}^{-}\right), P\left(\frac{3}{2}, \frac{3}{2}^{-}\right) \text {, } \\
& \bar{c} A_{(c q)} A_{\left(q^{\prime} q^{\prime \prime}\right)}\left(2^{+}\right): \quad P\left(\frac{1}{2}, \frac{3}{2}^{-}\right), P\left(\frac{1}{2}, \frac{5}{2}^{-}\right), P\left(\frac{3}{2}, \frac{3}{2}^{-}\right), P\left(\frac{3}{2}, \frac{5}{2}^{-}\right) \text {. }
\end{aligned}
$$

\subsubsection{Diquark-antidiquark mesons: masses and spin splitting parameter}

In Ref. 15 the diquark-antidiquark mesons, $(c q) \cdot\left(\bar{c} \bar{q}^{\prime}\right)$, were studied, we use this study as a guide for consideration of the pantaquarks.

The masses of the heavy diquarks were determined in Ref. 15, the masses of the light ones were determined in the previous studies. $\frac{3}{}$ The masses read:

$$
\begin{array}{ll}
m_{S\left(q^{\prime} q^{\prime \prime}\right)}=650 \pm 50 \mathrm{MeV}, & m_{A\left(q^{\prime} q^{\prime \prime}\right)}=750 \pm 50 \mathrm{MeV} \\
m_{S\left(s q^{\prime}\right)}=770 \pm 50 \mathrm{MeV}, & m_{A\left(s q^{\prime}\right)}=870 \pm 50 \mathrm{MeV} \\
m_{S(s s)}=900 \pm 50 \mathrm{MeV}, & m_{A(s s)}=1000 \pm 50 \mathrm{MeV} \\
m_{S(c q)}=2000 \pm 50 \mathrm{MeV}, & m_{A(c q)}=2050 \pm 50 \mathrm{MeV} \\
m_{S(c s)}=2100 \pm 50 \mathrm{MeV}, & m_{A(c s)}=2150 \pm 50 \mathrm{MeV}
\end{array}
$$


For a rough estimation of the tetraquark masses the mass-splitting formula was written in Ref. 15 as:

$$
M_{(c q) \cdot\left(\bar{c} \bar{q}^{\prime}\right)}^{(J)}=m_{(c q)}+m_{\left(\bar{c} \bar{q}^{\prime}\right)}+J(J+1) \Delta .
$$

The value $\Delta=70 \pm 10 \mathrm{MeV}$ fits the data. Within errobars the value for spin splitting parameter $\Delta$ coincides with that for $c \bar{c}$ mesons. Indeed, for $J / \psi$ and $\eta_{c}$ one has $m_{J / \psi}-m_{\eta_{c}} \simeq 120 \mathrm{MeV}$ that gives $\Delta_{c \bar{c}} \simeq 60 \mathrm{MeV}$. For estimation of masses of strange diquark-antidiquark states the same parameter $\Delta$ is used in Ref. 15 .

\subsection{Estimation of masses of the pentaquarks}

It was understood relatively long agd ${ }^{26} 28$ that the mass splitting of hadrons can be well described in the framework of the quark model by the short-ranged spin-spin interactions of the constituents. For mesons and baryons the mass formulae discussed by Glashow ${ }^{28}$ read:

$$
\begin{aligned}
& M_{M}=\sum_{j=1,2} m_{q(j)}+a \frac{\vec{s}_{1} \vec{s}_{2}}{m_{q(1)} m_{q(2)}}, \\
& M_{B}=\sum_{j=1,2,3} m_{q(j)}+b \sum_{j>\ell} \frac{\vec{s}_{j} \vec{s}_{\ell}}{m_{q(j)} m_{q(\ell)}}
\end{aligned}
$$

where $\vec{s}_{j}$ and $m_{q(j)}$ refer to spins and masses of the constituents. Mass splitting parameters in (8), $a$ and $b$, are characterized by a size of the color-magnetic interaction in the discussed hadron, the short-range interaction is supposed in Ref. 28. For the 36-plet mesons and 56-plet baryons formulae of Eq. (8) work well. The only exception is the pion, its calculated mass is $\sim 350 \mathrm{MeV}$ that point out an existence of additional forces in the pseudoscalar channel (possibly, istanton-induces forces, see Ref. 29).

Operating with mass-splitting term $J(J+1) \Delta$ we have for the light hadrons: $\Delta_{\Delta-N}=$ $67 \mathrm{MeV}, \Delta_{\omega-\eta}=115 \mathrm{MeV}$, and for charmonium mesons: $\Delta_{J / \psi-\eta_{c}}=60 \mathrm{MeV}$. For the

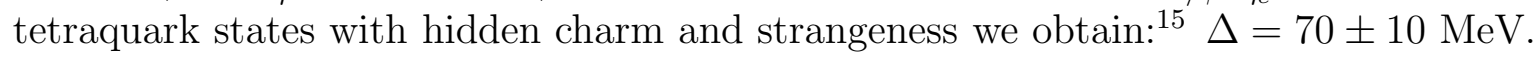

For evaluation of pentaquark masses we use similar procedure. First, let us estimate the masses of diquark-diquark subsystems $(c q) \cdot\left(q^{\prime} q^{\prime \prime}\right)$. For the diquark-diquark subsystem we write:

$$
M_{(c q) \cdot\left(q^{\prime} q^{\prime \prime}\right)}=m_{(c q)}+m_{\left(q^{\prime} q^{\prime \prime}\right)}+J_{(c q) \cdot\left(q^{\prime} q^{\prime \prime}\right)}\left(J_{(c q) \cdot\left(q^{\prime} q^{\prime \prime}\right)}+1\right) \Delta_{(c q) \cdot\left(q^{\prime} q^{\prime \prime}\right)},
$$

with the splitting parameter being of the order of that for the diquark-antidiquark system, $\Delta_{(c q) \cdot\left(q^{\prime} q^{\prime \prime}\right)} \sim 70 \mathrm{MeV}$. As the next step we write masses of the pentaquarks as a sum of masses of the constituents plus spin splitting term:

$$
\begin{aligned}
M_{\bar{c} \cdot(c q)\left(q^{\prime} q^{\prime \prime}\right)} & =m_{\bar{c}}+M_{(c q) \cdot\left(q^{\prime} q^{\prime \prime}\right)}+J_{\bar{c} \cdot(c q)\left(q^{\prime} q^{\prime \prime}\right)}\left(J_{\bar{c} \cdot(c q)\left(q^{\prime} q^{\prime \prime}\right)}+1\right) \Delta_{\bar{c} \cdot(c q)\left(q^{\prime} q^{\prime \prime}\right)} \\
& \simeq m_{\bar{c}}+M_{(c q) \cdot\left(q^{\prime} q^{\prime \prime}\right)}
\end{aligned}
$$

where the mass of the constituent antiquark $\bar{c}$ is equal to $m_{c}=1300 \pm 50 \mathrm{MeV} ! 31$ In the last line of Eq. (10) we neglect the mass splitting term, $\Delta_{\bar{c} \cdot(c q) \cdot\left(q^{\prime} q^{\prime \prime}\right)} \rightarrow 0$. The experimental data ${ }^{4}$ require this parameter to be small, $\Delta_{\bar{c} \cdot(c q) \cdot\left(q^{\prime} q^{\prime \prime}\right)}<15 \mathrm{MeV}$. We put it zero in order to manage without cumbersome terms. 
Following the Eq. (10) we write for the low-laying non-strange pentaquarks $P_{\bar{c} \cdot(c q)\left(q^{\prime} q^{\prime \prime}\right)}$ with $q, q^{\prime}, q^{\prime \prime}=u, d:$

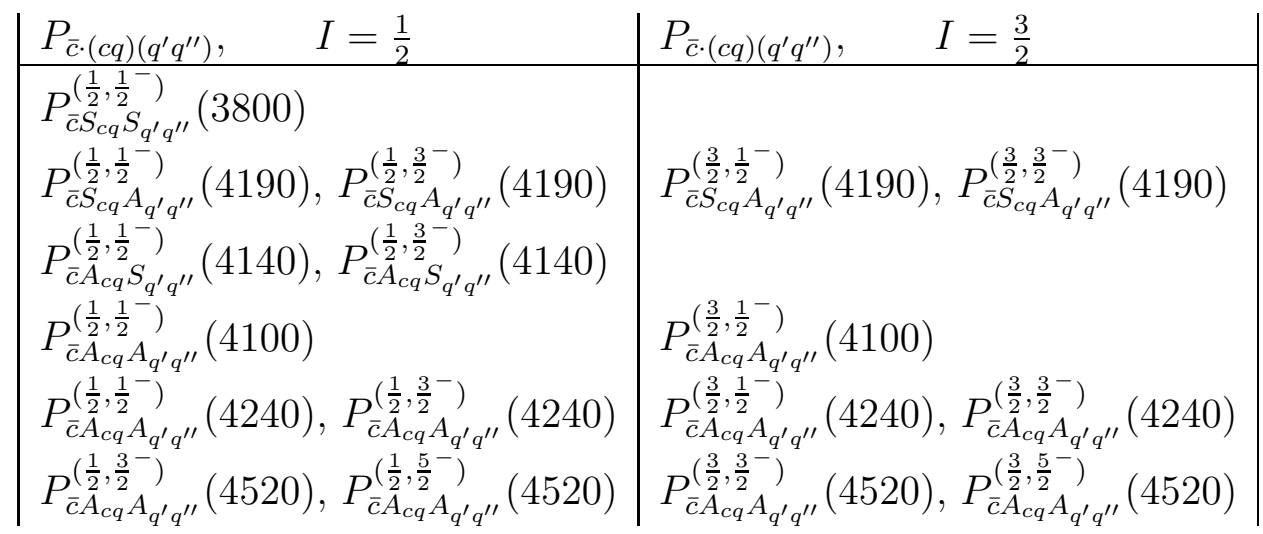

Masses are given in $\mathrm{MeV}$ units, the uncertainty in the determination of masses is of the order $\pm 150 \mathrm{MeV}$. We consider the state $P_{A_{(c q)}}^{\left(\frac{1}{2}, \frac{5}{2}{ }^{-}\right)} A_{\left(q^{\prime} q^{\prime \prime}\right)}(4520 \pm 150)$ as pentaquark seen in Ref. 4 which reveals itself as a narrow peak at $M_{p J / \psi}=4450 \mathrm{MeV}$ in the $(p J / \psi)$-spectrum.

\subsection{Pentaquarks with open strangeness}

We have two sets of pentaquark states $P_{\bar{c} \cdot(c q)\left(s q^{\prime}\right)}$ with open strangeness $S=1$ :

\begin{tabular}{|c|c|}
\hline$P_{\bar{c} \cdot(c q)\left(s q^{\prime}\right)}$ & $P_{\bar{c} \cdot(c q)\left(s q^{\prime}\right)}$, \\
\hline$P_{\bar{c} S_{c q} S_{s g^{\prime}}}^{\left(0, \frac{1}{2}\right)}(4070)$ & $P_{\bar{c} S_{c q} S_{s g^{\prime}}}^{\left(1, \frac{1}{2}^{-}\right)}(4070)$ \\
\hline 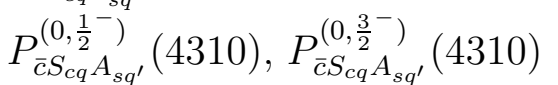 & $P_{\bar{c} S_{c q} A_{s q^{\prime}}}^{\left(1, \frac{1}{-}^{-}\right)}(4310), P_{\bar{c} S_{c q} A_{s q^{\prime}}}^{\left(1, \frac{3}{2} A^{-}\right)}(4310)$ \\
\hline$P_{\bar{c} A_{c q} S_{s q^{\prime}}^{(}}^{\left(0, \frac{1}{2}^{-}\right)}(4260), P_{\bar{c} A_{c q} S_{s q^{\prime}}}^{\left(0, \frac{3}{2^{-}}\right)^{\prime}}(4260)$ & $P_{\bar{c} A_{c q} S_{s q^{\prime}}}^{\left(1, \frac{1}{}^{-}\right)^{-}}(4260), P_{\bar{c} A_{c q} S_{s q^{\prime}}}^{\left(1, \frac{3}{2}\right)^{-}}(4260)$ \\
\hline$P_{\bar{c} A_{c q} A_{s q^{\prime}}}^{\left(0, \frac{1^{-}}{2}\right)}(4220)$ & $P_{\bar{c} A_{c q} A_{s q^{\prime}}}^{\left(1, \frac{1}{2}^{-}\right)}(4220)$ \\
\hline$P_{\bar{c} A_{c q} A_{s q^{\prime}}}^{\left(0, \frac{1}{}^{-}\right)^{s q}}(4360), P_{\bar{c} A_{c q} A_{s q^{\prime}}}^{\left(0, \frac{3}{2}^{-}\right)}(4360)$ & $P_{\bar{c} A_{c q} A_{s q^{\prime}}}^{\left(1, \frac{1}{1}^{-}\right)}(4360), P_{\bar{c} A_{c q} A_{s q^{\prime}}}^{\left(1, \frac{3}{2}^{-}\right)}(4360)$ \\
\hline$P_{\bar{c} A_{c q} A_{s q^{\prime}}}^{\left(0, \frac{3}{2^{-}}\right)}(4640), P_{\bar{c} A_{c q} A_{s q^{\prime}}}^{\left(0, \frac{5}{2}^{-}\right)}(4640)$ & $P_{\bar{c} A_{c q} A_{s q^{\prime}}}^{\left(1, \frac{3}{2}{ }^{-}\right)}(4640), P_{\bar{c} A_{c q} A_{s q^{\prime}}}^{\left(1, \frac{5}{2}^{-}\right)}(4640)$ \\
\hline
\end{tabular}

and $P_{\bar{c} \cdot(c s)\left(q q^{\prime}\right)}$ with $S=1$ :

\begin{tabular}{|c|c|}
\hline$P_{\bar{c} \cdot(c s)\left(q q^{\prime}\right)}$ & $P_{\bar{c} \cdot(c s)\left(q q^{\prime}\right)}$ \\
\hline 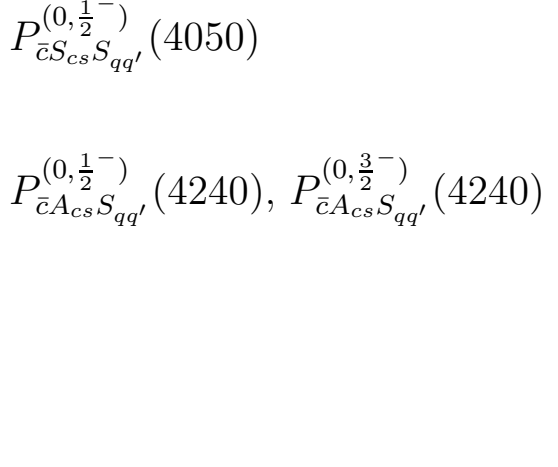 & $\begin{array}{l}P_{\bar{c} S_{c s} A_{q q^{\prime}}}^{\left(1, \frac{1}{2}^{-}\right)}(4290), P_{\bar{c} S_{c s} A_{q q^{\prime}}}^{\left(1, \frac{3}{2}{ }^{-}\right)}(4290) \\
P_{\bar{c} A_{c s} A_{q q^{\prime}}}^{\left(1, \frac{1}{2}^{-}\right)}(4200) \\
P_{\bar{c} A_{c s} A_{q q^{\prime}}}^{\left(1, \frac{1}{2}^{-}\right)}(4340), P_{\bar{c} A_{c s} A_{q q^{\prime}}}^{\left(1, \frac{3}{2}^{-}\right)}(4340) \\
P_{\bar{c} A_{c s} A_{q q^{\prime}}}^{\left(1, \frac{3}{2}{ }^{-}\right.}(4620), P_{\bar{c} A_{c s} A_{q q^{\prime}}}^{\left(1, \frac{5}{2}^{-}\right)}(4620)\end{array}$ \\
\hline
\end{tabular}

In this sector we see practically overlapping states with equal $(I, J)$ values, like, e.g.,

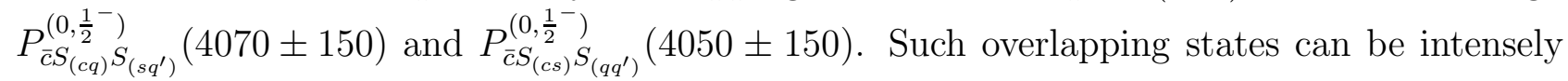


intermix so that one of the states accumulates the width of the other ones ${ }^{32}$ - as a result we see a narrow peak on a broad substrate.

A set of the pentaquark states $P_{\bar{c} \cdot(c q)(s s)}$ and $P_{\bar{c} \cdot(c s)(s q)}$ with strangeness $S=2$ can be written as:

\begin{tabular}{|c|c|}
\hline$P_{\bar{c} \cdot(c q)(s s)}$ & $P_{\bar{c} \cdot(c s)(s q)}$ \\
\hline$P_{\bar{c} S_{c q} S_{s s}}^{\left(\frac{1}{2}, \frac{1}{2}-\right)}(4200)$ & $P_{\bar{c} S_{c s} S_{s q}}^{\left(\frac{1}{2}, \frac{1}{2}{ }^{-}\right)}(4170)$ \\
\hline$P_{\bar{c} S_{c q} A_{s s}}^{\left(\frac{1}{2}, \frac{1}{2}{ }^{-}\right)}(4440), P_{\bar{c} S_{c q} A_{s s}}^{\left(\frac{1}{2}, \frac{3}{2}{ }^{-}\right)}(4440)$ & $P_{\bar{c} S_{c s} A_{s q}}^{\left(\frac{1}{2}, \frac{1}{2}^{-}\right)}(4410), P_{\bar{c} S_{c s} A_{s q}}^{\left(\frac{1}{2}^{2}, \frac{3}{}^{-}\right)}(4410)$ \\
\hline$P_{\bar{c} A_{c q} S_{s s}}^{\left(\frac{1}{2}, \frac{1}{2}-\right)}(4390), P_{\bar{c} A_{c q} S_{s s}}^{\left(\frac{1}{2}, \frac{3}{2}-\right)}(4390)$ & $P_{\bar{c} A_{c s} S_{s q}}^{\left(\frac{1}{2}, \frac{1}{2}{ }^{-}\right)}(4460), P_{\bar{c} A_{c s} S_{s q}}^{\left(\frac{1}{2}, \frac{3}{2}{ }^{-}\right)}(4460)$ \\
\hline$P_{\bar{c} A_{c q} A_{s s}}^{\left(\frac{1}{2}, \frac{1}{2}\right)}(4350)$ & $P_{\bar{c} A_{c s} A_{s q}}^{\left(\frac{1}{2}, \frac{1}{}^{-}\right)}(4320)$ \\
\hline 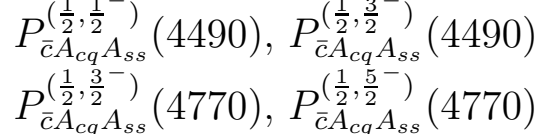 & 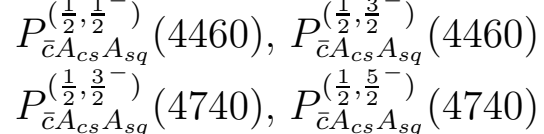 \\
\hline
\end{tabular}

In this sector we also see nearly overlapping states.

\subsection{The LHCb data: where are other exotic states?}

The LHCb peak $5 / 2^{?}(4450 \pm 4)$ being interpreted as pentaquark state arises questions. Existence of pentaquark means an existence of an assembly of pentaquarks. Specifically, the pentaquark with $J^{P}=5 / 2^{-}$should be accompanied by states with smaller masses and $J^{P}=3 / 2^{-}, 1 / 2^{-}$, see Eq. (11); the similar assembly exists for positive parity pentaquarks. The multiplet neighbours are not observed in Ref. 4. Special studies are needed to clarify if these assemblies of states do realized or not.

Moreover, if pentaquark states exist the tetraquark states exist as well. It means that in the $K J / \psi$ sector diquark-antidiquark states $(c s) \cdot(\bar{c} \bar{d})$ can be observed in the mass region 4000-4500 $\mathrm{MeV}$, production of the low-lying tetraquarks is not forbidden in the decay $\Lambda_{b}^{0} \rightarrow K^{-} J / \psi p$. But definite indication for production of such states is absent in Ref. 4. Production of the tetraquark states $(c s) \cdot(\bar{c} \bar{d})$ in the decay $\Lambda_{b}^{0} \rightarrow K^{-} J / \psi p$ would mean crossing of tetraquark and petraquark signals on Dalitz-plot thus complicating interpretation of the data.

\section{Conclusion}

The scheme of the low-lying S-wave pentaquarks is developed on the basis of the study of the tetraquark states $^{15}$ and results of the LHCb-collaboration. ${ }^{4}$ Following Ref. 16 we suggest that the narrow peak $5 / 2^{?}(4450 \pm 4)$ with $\Gamma=(39 \pm 24) \mathrm{MeV}$ presents the $S$-wave pentaquark denoted here as $P_{S_{(c q)}}^{\left(\frac{1}{2}^{\frac{5}{2}} A_{\left(q^{\prime} s\right)}^{-}\right)}(4520 \pm 150)$, see Eq. (11). Than the broad bump, $3 / 2^{?}(4380 \pm 38)$ with effective width $\Gamma=(205 \pm 94) \mathrm{MeV}$, also seen in the $(p J / \psi)$ spectrum, can be related with possible resonances of the $K^{-} J / \psi$-channel located in the mass region $4000-4500 \mathrm{MeV}$.

According to the diquark-diquark-antiquark scheme the mass interval 4040-4500 MeV contains several exotic baryon resonances with $J^{P}=3 / 2^{-}, 1 / 2^{-}$. Interplay of these baryon states with exotic mesons from the $K^{-} J / \psi$-channel may be crucial for decipher the Dalitz-plot. 
Search for exotic baryon resonances in other channals may be effective, for example, decay of the low-laying $1 / 2^{-}$-states into the $\left(p \eta_{c}\right)$-channel looks as dominant.

\section{Acknowledgments}

The work was supported by grant RSCF-14-22-00281.

\section{References}

[1] M. M. Gell-Mann, Phys. Lett. 8, 214 (1964).

[2] A.V. Anisovich, V.V. Anisovich, M.A. Matveev, V.A. Nikonov, J. Nyiri, A.V. Sarantsev Mesons and Baryons, (World Scientific, Singapore, 2008).

[3] A.V. Anisovich, V.V. Anisovich, M.A. Matveev, V.A. Nikonov, A.V. Sarantsev and T.O. Vulfs, Int. J. Mod. Phys. A25, 2965 (2010) [Phys. Atom. Nucl. 74, 418 (2011)].

[4] The LHCb collaboration, arXiv:1507.03414v1 [hep-ex].

[5] R.L. Jaffe, Phys. Rev. D 15, 281 (1977).

[6] A. De Rujula, H. Georgy, S.L. Glashow, Phys. Rev. Lett. 38, 317 (1977).

[7] D. Strottman, Phys. Rev. D 20, 748 (1979).

[8] L.Maiani, F.Piccinini, A.D.Polosa, V.Riquer, Phys. Rev. D 71, 014028 (2005).

[9] M.B. Voloshin, Phys. Rev. D 84, 031502 (2011).

[10] A. Ali, C. Hambrock, W.Wang, Phys. Rev. D 85, 054011 (2012).

[11] S. Weinberg, Phys. Rev. Lett. 110, 261601 (2013).

[12] S.J. Brodsky, D.S. Hwang and R.F. Lebed Phys. Rev. Lett. 113, 112001 (2014).

[13] R.L. Jaffe and F. Wilchek, Phys. Rev. Lett. 91, 232003 (2003).

[14] G.C. Rossi and G. Veneziano, Phys. Lett. B 597, 338 (2004).

[15] V.V. Anisovich, M.A. Matveev, A.V. Sarantsev, A.N. Semenova, arXiv:1507.7232 [hep-ph].

[16] V.V. Anisovich, M.A. Matveev, J. Nyiri, A.V. Sarantsev, A.N. Semenova, arXiv:1507.07652 [hep-ph].

[17] L. Maiani, A.D. Polosa, and V. Riquer arXiv:1507.04980v1 [hep-ph].

[18] V.V. Anisovich, M.A. Matveev, J. Nyiri, A.V. Sarantsev, A.N. Semenova, arXiv:1509.03028[hep-ph]. 
[19] M. Mikhasenko, arXiv:1507.06552v1 [hep-ph].

[20] X.-H. Liu, Q. Wang and Q. Zhao, arXiv:1507.05359v1 [hep-ph].

[21] U.-G. Meissner and J.A. Oller, arXiv:1507.07478v1 [hep-ph].

[22] A. Mironov and A. Morozov, arXiv:1507.04694v1 [hep-ph].

[23] J. He, arXiv:1507.05200v1 [hep-ph].

[24] R.F. Lebed, arXiv:1507.05867v1 [hep-ph].

[25] F.-K. Guo, U.-G. Meißner, W. Wang and Z. Yang, arXiv:1507.04950 [hep-ph].

[26] Y.B. Zeldovich, A.D. Sakharov, Yad. Fis. 4, 395 (1966).

[27] A. de Rujula, H. Georgi, S.L. Glashow, Phys. Rev. D 12, 147 (1975).

[28] S.L. Glashow, Particle physics far from high energy frontier, (Harvard Preprint, HUPT80/A089, 1980).

[29] E.V. Shuryak, Nucl. Phys. B 203, 93 (1982).

[30] V.V. Anisovich, L.G. Dakhno, M.A. Matveev, V.A. Nikonov and A.V. Sarantsev, Yad. Fiz. 70, 392 (2007) [Phys. Atom. Nucl. 70, 364 (2007)].

[31] A.V. Manohar, C.T. Sachrajda, Phys. Rev. D 66, 010001 (2002).

[32] V.V. Anisovich, D.V. Bugg and A.V. Sarantsev, Phys. Rev. D 58, 111503 (1998). 